\title{
Semantic disturbance in schizophrenia and its relationship to the cognitive neuroscience of attention
}

\section{Citation}

Nestor, P.G, S.D Han, M Niznikiewicz, D Salisbury, K Spencer, M.E Shenton, and R.W McCarley. 2001. Semantic Disturbance in Schizophrenia and Its Relationship to the Cognitive Neuroscience of Attention. Biological Psychology 57, no. 1-3: 23-46. doi:10.1016/s0301-0511(01)00088-6.

\section{Published Version}

doi:10.1016/s0301-0511(01)00088-6

\section{Permanent link}

http://nrs.harvard.edu/urn-3:HUL.InstRepos:28520165

\section{Terms of Use}

This article was downloaded from Harvard University's DASH repository, and is made available under the terms and conditions applicable to Other Posted Material, as set forth at http:// nrs.harvard.edu/urn-3:HUL.InstRepos:dash.current.terms-of-use\#LAA

\section{Share Your Story}

The Harvard community has made this article openly available.

Please share how this access benefits you. Submit a story.

\section{Accessibility}


Biol Psychol. 2001 ; 57(1-3): 23-46.

\title{
Semantic disturbance in schizophrenia and its relationship to the cognitive neuroscience of attention
}

\author{
P.G. Nestor ${ }^{\star}$, S.D. Han, M. Niznikiewicz, D. Salisbury, K. Spencer, M.E. Shenton, and R.W. \\ McCarley \\ Department of Psychiatry, Harvard Medical School, Brockton VAMC-116A, 940 Belmont Street, \\ Brockton, MA 02401, USA
}

\begin{abstract}
We view schizophrenia as producing a failure of attentional modulation that leads to a breakdown in the selective enhancement or inhibition of semantic/lexical representations whose biological substrata are widely distributed across left (dominant) temporal and frontal lobes. Supporting behavioral evidence includes word recall studies that have pointed to a disturbance in connectivity (associative strength) but not network size (number of associates) in patients with schizophrenia. Paralleling these findings are recent neural network simulation studies of the abnormal connectivity effect in schizophrenia through 'lesioning' network connection weights while holding constant network size. Supporting evidence at the level of biology are in vitro studies examining $N$-methylD-aspartate (NMDA) receptor antagonists on recurrent inhibition; simulations in neural populations with realistically modeled biophysical properties show NMDA antagonists produce a schizophrenialike disturbance in pattern association. We propose a similar failure of NMDA-mediated recurrent inhibition as a candidate biological substrate for attention and semantic anomalies of schizophrenia.
\end{abstract}

\section{Keywords}

Schizophrenia; Attention; NMDA; Inhibition; Neural network

\section{Introduction}

In the early 1900s, pioneering figures of modern psychopathology no less than Kraepelin and Bleuler each emphasized neuropsychological disturbance as central to schizophrenia. As contemporaries of Alois Alzheimer, both Kraepelin and Bleuler presumed that these problems in information processing originated from brain pathology that would be ultimately revealed by future generations of researchers as has been the case in the type of dementia now known as Alzheimer's disease.

Kraepelin's writings described a number of disease-related neuropsychological problems, none though more central than that which clearly echoes contemporary neuroscience models of attention (Parasuraman, 1997). In his classic, Dementia Praecox and Paraphrenia, Kraepelin wrote of what today may be viewed as a failure to regulate attention, which he described as follows:

"It is quite common for them to lose both inclination and ability on their own initiative to keep their attention fixed for any length of time. The patients digress, do not stick

(C) 2001 Elsevier Science B.V. All rights reserved.

*Corresponding author. Fax: +1-508-5860894. paul.nestor@umb.edu (P.G. Nestor). 
to the point, let their thoughts wander without voluntary control in the most varied directions. On the other hand, the attention is often rigidly fixed for a long time, so that the patients stare at the same point, or at the same object, continue the same line of thought, or do not let themselves be interrupted in some definite piece of work. Further it happens that they deliberately turn away their attention from those things to which it is desired to attract it, turn their backs when spoken to and turn their eyes if anything is shown to them. But in the end, there is occasionally noticed a kind of irresistible attraction to casual external impressions."

Bleuler wrote of the famous four A's of schizophrenia (ambivalence, autism, affect, associations), prominent among these was a disturbance of associations reflected in a breakdown in putative associative threads that serve to interweave words, thoughts, and ideas into coherent discourse. Here Bleuler captured an aspect of schizophrenic cognition that has almost become specific if not unique to the disease-related disturbance of thought that is rarely reported in neurological conditions resulting from trauma or degenerative processes. Bleuler maintained that schizophrenic discourse is often contaminated by associative intrusions, as evidenced by the famous example of his patient identifying her family members as 'father, son, and Holy Ghost'. Associative disturbance would become a striking feature of schizophrenia and related psychotic conditions, with psychometric measures now commonly used to assess schizophrenic thought, the most notable example being the Thought Disorder Index (TDI) developed by Holzman and colleagues (Johnston and Holzman, 1979). Consider the following excerpt from the TDI of a patient with schizophrenia without, important to note, any formal neurological disturbance in language:

Interviewer: "Tell me about school"

Patient: "Well there are schools of play and schools of fish, mostly you see fish school, people edumacating themselves, you see, sea is one thing and education is another. Fish is school in their community, that's why the community of man stands in the way of community of fish..."

Striking is the resonance of these now classic observations with contemporary neuropsychological models that have emerged from the burgeoning new field of cognitive neuroscience, with its central tenet being the anatomization of cognition into discrete operations and processes linked to selective properties and functions of neural systems and networks. These prescient observations point to the important role of two broad domains of cognition, attention and language, in the neuropsychological disturbance of schizophrenia. Each domain subsumes a diverse array of codes, processes, rules, and structures, so that to study the interaction of disturbances in language and attention in schizophrenia, which itself is also known for its heterogeneous presentation, requires a number of points of clarification. We thus begin by examining briefly cognitive neuroscience studies of attention, followed by a similarly brief examination of language, limited to the semantic language system that represents the conceptual content (the meaning) of words, thoughts, sentences, images, and beliefs. We will then review experimental evidence relevant to attention and semantic disturbances in schizophrenia from a number of diverse methodologies of cognitive neuroscience, ranging from basic cellular biology to event-related potentials (ERPs), and extending to simulations of artificial neural networks. For heuristic consideration, we will examine these experimental findings within a conceptual framework that bears on the problem of attention and semantics in the neuropsychology of schizophrenia.

\section{Neuroscience of attention}

Among the most influential views on attention are those that emphasize its selective and modulatory function. In the past decade, several neuroimaging studies of both animals and humans demonstrated selective and specific attention effects on neuronal circuits (e.g. Moran 
and Desimone, 1985; Ramachadran and Cobb, 1995; Britten, 1996; Treue and Maunsell, 1996; Rees et al., 1997). These studies have largely shown that attention selectively modulates neuronal response by either facilitating or sharpening firings of already activated cells (e.g. Spitzer et al., 1988) or by suppressing or inhibiting neighboring deactivated cells (e.g. Moran and Desimone, 1985).

These attention effects are distinct from those that might be linked to arousal. Attention unlike arousal - modulates only those circuits that are activated in response to specific information processing demands, as for example stimulus encoding or perceptual discrimination. It, therefore, exerts a specific effect unlike the nonspecific effect of arousal. Moreover, attention effects extend beyond neuronal circuitry to brain networks and systems that support high-level cognition. As cognition become more intensive or demanding, attention is called to help drive the computational machinery. For example in a functional MRI study of sentence comprehension, Just et al. (1996) demonstrated that the increasing linguistic complexity of sentences produced a proportionate increase of activation of the classical left hemisphere language system in healthy controls. By the same token, processes that are presumed automatic and involuntary may also be effected, with attention disregulation perhaps leading to states of hyper-automaticity (Callaway and Naghdi, 1982).

In this sense, then, attention operates in terms of a modulatory or gain function that increases the strength of selected sensory input and decreases the strength of unselected sensory input (Nestor and O'Donnell, 1997). Often occurring early in the information-processing stream within 100 ms post-stimulus (e.g. Hillyard et al., 1995), these attention effects may be widely distributed throughout the brain, although much more common in secondary and tertiary cortical areas than primary areas. For example, for visual stimuli, modulatory effects are seen in extrastriate but not primary cortical areas (Colby, 1991; Corbetta et al., 1991; Haxby et al., 1991), and in temporal but not brainstem areas for auditory stimuli (Hillyard et al., 1995)

Attention effects might thus transverse three levels of neural organization: circuits, networks, and systems, with each level differing in terms of scale, content, and function. At the most basic level, attention is seen as enhancing signal processing of local neuronal circuits by constraining and amplifying content-free activation. Bound by both time and space, these local circuits are organized to form distributed networks that support symbol processing. Network communication is facilitated by attention, which is often represented and simulated by idealized synapses of connectionist models (e.g. Shastri and Ajjanagadde, 1993). These networks in turn help form wide-scale neural computational systems of perception, memory, language and other higher-order cognitive modules in which the role of attention is to insure the fidelity of computational contents (Mesulam, 1990). Thus in this model, brain and attention are composed of constituent operations and processes that are supported by discrete local circuits that act in concert to form widely-distributed networks and large-scale systems. Attention and its derivative cognition emerge as a product of intricate relationships between and among brain circuits, networks, and systems, each of which may be modulated and reconfigured by dictates of intrinsic and extrinsic neurotransmitters (Robbins, 1997). These attention effects are evident by the selective enhancement or inhibition of neuronal response (Nestor and O'Donnell, 1997).

Theses attention processes serve to deliver selected signals or input to higher cognitive centers. The emphasis is on signal or informational selectivity, generally considered to be the essence of attention (Posner, 1988). However, other neuronal properties in addition to the selective enhancement or inhibition of firing rates of neurons have been proposed as important to promoting states of attention in the brain (Crick and Koch, 1990; Niebur and Koch, 1997). For example, a computational model of visual attention (Niebur and Koch (1997), p. 178) proposed a temporal tagging hypothesis in which attentional selection "works by marking (or tagging) 
the selected stimuli, based on the temporal fine structure of neuronal spike trains. Only such tagged stimuli can access awareness and memory, and only those stimuli will contribute to the decision making process leading to motor actions".

Niebur and Koch (1997) specifically proposed as a neural mechanism of tagging synchronicity of spiking neurons in response to stimulus-dependent firing rates. They compared the temporal structure of spike trains of neurons from receptive fields (e.g. V2) falling within the focus of attention with those that fell outside the focus of attention. Their results indicated that for spike trains of neurons with receptive fields that did not include the attended stimulus, a distribution of cell firings emerged that reflected a stochastic process known as a homogeneous Poisson process, described as 'memoryless' although each spike showed a refractory period. By contrast, spike trains of neurons with receptive fields within the focus of attention showed a probabilistic distribution, reflected by the correlation (or synchronization) among spike trains responding to an attended stimulus. Niebur and Koch (1997) proposed that such tagging occurs within the gamma or $40 \mathrm{~Hz}$. range, leading to the segregation of attended stimuli and their coincident, synchronous, and correlated spike trains of neurons from unattended stimulus with their asynchronous, uncorrelated patterns of neuronal firing.

To summarize, cognitive neuroscience studies have examined the spatial-temporal distribution and dynamics of attention within the brain. As will be discussed below, and already briefly addressed above, attention as a modulation of specific neuronal response via neurotransmission represents a very appealing and powerful heuristic model to examine schizophrenic cognition. Likewise, the extent to which attention effects are linked to synchronous or coordinated firings of spike trains of neurons may also have special relevance to understanding schizophrenic cognition. This is highlighted by recent EEG evidence from our laboratory pointing to a schizophrenia-related failure of entrainment in response to $40 \mathrm{~Hz}$ but not 20 or $30 \mathrm{~Hz}$ auditory clicks (Kwon et al., 1999). This represents the same gamma range that Niebur and Koch (1997) demonstrated in their computational simulations to be the basis of attention tagging of stimuli for further processing. However, before discussing evidence from studies of schizophrenic patients, we will now address briefly cognitive neuroscience studies of semantic knowledge in relation to attention in order to provide additional theoretical scaffolding for examining schizophrenic cognition.

\section{Attention and semantic processes}

Models of language assume the existence of a mental lexicon or brain dictionary that is widely distributed across frontal and temporal lobes (Carmazza, 1996). Words are represented as networks of interconnected nodes, with either a one-to-one representation of a word and a node or a distributed representation of various word features and selected sets of nodes. Presentation of a word activates its lexical representation, with activation spreading automatically or obligatorily to other related lexical entries (Collins and Loftus, 1975; Neely, 1976). Various word features, such as orthographic, phonological and semantic are represented in the lexicon, but the most powerful determinant of processing are semantic representations of concepts of corresponding words, which is the heart of language (Fischler, 1997). Lexical and semantic processes are separable, with the former generally confined to word/non word representations and the latter to word meanings.

Each may also be supported by different brain regions. For example, at the lexical level, Nobre and McCarthy (1994) compared evoked related potentials, as assessed by intracranial recording, to visual words presented singly and to orthographically illegal non-words. They demonstrated that visual words produced large negative field potentials within the anterior portion of the left medial temporal lobe, in relation to orthographically illegal non-words. In a similar vein, neurological studies have shown that naming failures that are lexical in nature 
occur when a patient can describe and define an object, thus providing a semantic meaning of it, yet fail to produce its specific name (Carmazza, 1996; Damassio et al., 1996). Imaging studies as well as anatomical correlations have pointed to left temporal lobe involvement in lexical representation (Damassio et al., 1996). By contrast, structures of the left and right hemispheres support the distributed sets of conceptual features that provide meaning to words, and these features are thought to be collected in the left temporal lobe for further lexical processing and, ultimately, for speech production (Carmazza, 1996).

In patients with schizophrenia, a number of independent lines of evidence has pointed to abnormalities in brain regions very near if not overlapping with those identified as converging areas for lexical representation (Damassio et al., 1996). In particular, MRI studies of patients with schizophrenia have revealed reduced temporal lobe volume, including medial temporal lobe and posterior superior temporal gyrus (McCarley et al., 1999). Paralleling these findings of structural brain abnormalities in left temporal areas thought to be intimately involved in lexical and semantic processing are ERP studies demonstrating auditory P300 reduction that are greater at left compared to right electrode sites in schizophrenia (McCarley et al., 1997). In addition, McCarley et al. (1993) demonstrated that P300 amplitude at left temporal electrode site correlated significantly with reduced STG volume, as measured by quantitative MRI, in the same group of patients with schizophrenia. And perhaps most interesting in terms of a potential neurobiological substrate for schizophrenic thought disorder are findings linking reduced STG to an objective clinical measure of severity of thought disturbance, as assessed by the TDI (Shenton et al., 1992).

These studies help to identify semantic and lexical structures, but perhaps what is most interesting is how attention processes interact with these semantic structures to promote efficient cognition as well as to how they may be compromised by schizophrenia leading to disordered cognition. Consider that in healthy cognition attention interacts with semantic and lexical representation, often by either facilitating or inhibiting processing. Attention directed to semantic aspects of words generally facilitates memory, whereas attention directed to perceptual attributes of words, such as their spelling impairs explicit memory (Craik and Tulving, 1975). Perhaps even more relevant to the current review is that the spread of activation within the semantic system is largely determined by attention. Activation spreads from one neighboring node to another, passively and automatically for approximately $500 \mathrm{~ms}$ poststimulus, without awareness and attention or at least controlled, voluntary attention (Posner and Snyder, 1975; Neely, 1977; den Heyer et al., 1983). Yet these facilitatory effects (priming) can be enhanced even further by directed attention (Posner and Snyder, 1975).

For example, semantic priming occurs when the processing of a target word (e.g. nurse) is facilitated by semantically related word (e.g. doctor) presented prior to the target as a prime. By contrast, semantic priming is eliminated by diverting attention away from the lexical level of words by having subjects perform a letter matching task on the prime words (Besner et al., 1990). Moreover, ignored stimuli, including words, have often been assumed to draw little or no attention. However, over the past 15 years, a phenomenon known as negative priming has been demonstrated, as best reflected by a decrement in processing prior distractors-turnedtargets in relation to other novel targets (e.g. Yee, 1991; Neill and Valdes, 1992). These studies have suggested that both excitation and inhibition operate to produce semantic priming.

Both semantic priming and negative priming represent important experimental paradigms that might shed light on information processing mechanisms underlying schizophrenic cognition. Several priming studies that might be viewed as derived from activation based models of cognition (see Anderson and Spellman, 1995; Nestor et al., 1998) have suggested that schizophrenia might be characterized by aberrant spread of automatic activation within semantic networks, leading to loose associations and derailed thinking, hallmark clinical 
indicators of schizophrenic thought disturbance (e.g. Firth, 1979; Spitzer et al., 1993; Nestor et al., 1997). Supporting the aberrant spread of automatic activation hypothesis are semantic priming studies that have demonstrated enhanced priming effects in patients with schizophrenia in relation to control (Manschreck et al., 1988; Spitzer et al., 1994) and psychiatric contrast groups (Kwapil et al., 1990). These effects, however, may be limited to relatively short onset asynchrony (SOA) times of less than $500 \mathrm{~ms}$.

On the other hand, using similar semantic priming experiments that sampled a range of SOA times, Barch et al. (1996) failed to demonstrate 'hyper-priming' effects in schizophrenia for SOAs ranging from very short (i.e. $250 \mathrm{~ms}$ ) to relatively long (i.e. $950 \mathrm{~ms}$ ). In fact, at long SOA $(950 \mathrm{~ms})$, the findings of Barch et al. demonstrated reduced priming in schizophrenic subjects. These data thus failed to support abnormality of automatic spread of activation within the lexical/semantic level of representation. Rather, Barch and colleagues interpreted their findings as evidence of 'hypo-priming' in schizophrenia, attributable to problems in attention controlled processes, which occur later (typically beyond $500 \mathrm{~ms}$ post stimulus) in the information processing stream, at the level of contextual representation.

Negative priming and related studies of perceptual processes (Beech and Clarridge, 1987; Beech et al., 1989; Elkins and Cromwell, 1994; Salo et al., 1997) have provided evidence of reduced negative priming in subjects with schizophrenia and related disorders. The basic idea behind negative priming is that the buildup of inhibition to a previous-distractor-turned target slows response time. Schizophrenic reduction in negative priming is therefore thought to reflect primarily faulty or reduced inhibition of prior distractors (Nestor and O'Donnell, 1997). In contrast, mechanisms underlying semantic priming abnormalities in schizophrenia have yet to be elucidated, although a combination of automatic and controlled attention processes are likely involved. Moreover, the relationship between semantic and negative priming has yet to be examined in schizophrenia. However, using a Stroop task, Salo et al. (1997) demonstrated not only reduced negative priming in a group of unmedicated outpatients with schizophrenia, but also showed evidence that some patients actually responded faster to those stimuli that were prior distractors. This latter pattern of performance resembled a hyper-priming effect demonstrated in several of the semantic priming studies discussed above.

To summarize, attention and semantic processes are intimately connected, although distinguishable experimentally, each by corresponding sets of neural representation. Attention effects are seen in either facilitation or inhibition of lexical/semantic structures that have been frequently demonstrated to be involved in the neuropathology and neurophysiology of schizophrenia. Perhaps even more telling is that the spread of activation within these lexical and semantic structures is largely thought to be governed by attention processes. Priming studies provide a means to assess spread of semantic activation as well as to index the more general inhibitory attention, as in the case of negative priming. Several studies, when taken together, have shown priming abnormalities in schizophrenia that do not fit with a generalized nonspecific impairment that has so often been the case with other behavioral findings of the past century (see Chapman and Chapman, 1973). These and the other foregoing studies thus provided a rationale for examining schizophrenic information processing disturbance as reflecting faulty interaction between attention and semantic processes. In the following section, we will present a tentative heuristic, top-down descriptive model of schizophrenic information processing abnormalities beginning with connectionist word (behavioral) studies, followed by a preliminary computer simulation of schizophrenic connectionist performance, concluding with a biophysical simulation of a proposed schizophrenic neural circuit model of failed cellular recurrent inhibition due to NMDA blockade. Each of these areas represents very distinct levels of analyses, ranging from behavioral to cellular. We seek to establish converging lines of experimental evidence among these levels to buttress an admittedly simplified and descriptive 
model of schizophrenic cognition. However, we do so for heuristic reasons, mindful if not skeptical of the pitfalls in cultivating such linkages.

\section{Connectionism and schizophrenic attention and semantic disturbances}

Connectionism is a school of cognitive science known for models of cognition that are typically referred to as neural networks due in large part to their massively parallel architectures and dynamic processing flexibility that are thought to resemble important elements of brain processing structures. In its simplest and most generic form, a neural network model consists of a number of interconnected units typically referred to as nodes, each with an activation value that is modified by connection weights. A model may be evaluated on a number of criteria, but none perhaps more relevant than the extent to which its architecture and accompanying processing characteristics approximate known biological properties and functions of the brain. The more biologically plausible are models that have simulated a specific characteristic or sets of characteristics of real neurons, such as temporal structure of cell firings in $\mathrm{V} 4$ of the macaque (Niebur et al., 1993) or antagonism of NMDA-mediated cellular recurrent inhibition (Grunze et al., 1996). The simulated characteristics are presumed to capture a fundamental property that is critical to the function of that modeled network of real neurons. Plausibility will be determined as to whether a network simulation, which by reason of the complexity of the brain is necessarily restricted to a finite set of characteristics, retains the functional properties of large biological networks of real neurons.

More common though are connectionist models that are psychological abstractions of human performance, such as associative memory. These models represent psychological phenomena based on some general principles of neural architecture and processing, but far less constrained in the absence of direct biological evidence of actual properties and functions of networks of real neurons. These models typically assume either a distributed or localist representation. In a localist representation, each node has a one-to-one correspondence to a symbolic referent, such as a word. In a distributed representation, no one particular node will be symbolic of a stimulus; various elemental features of a stimulus will be coded across nodes and recruited in processing other stimuli with shared or overlapping features. In either representation, nodes are organized into networks based on some index of presumed similarity of nearest neighbors, as for example groups of strongly associated words in models of associative memory. The effect of damage differs, however, for localist versus distributed models. Damage in a localist representation is often described as catastrophic, all or none, leading to the permanent disengagement and elimination of the disabled or 'lesioned' node. In contrast, damaging any single node may have a little or no effect in a distributed representation, and sustained damage that spreads to other nodes is thought to degrade performance.

In connectionist models, important aspects of attention and semantic processing can be captured by examining behavioral data collected with both control subjects and patients with schizophrenia (e.g. Cohen and Servan-Schreiber, 1992). In fact, in connectionist models that are intended to emulate information processing characteristics of distributed neural networks, attention is thought to modulate weights, causing a shift in responsiveness or activation values of units in competing processing pathways (Williams et al., 1996). In these models, units represent various features of words organized in networks of interconnected nodes, with each node corresponding to an idealized neuron (or, as we think more realistically, to a set of neurons), and each link (connection weight) corresponding to an idealized synaptic weight. As will be discussed below, once a schizophrenic behavioral pattern of performance can be established (Nestor et al., 1998), a connectionist model can be developed to test specific hypotheses related to underlying attention and semantic mechanisms that are predicted to contribute significantly to the schizophrenic behavioral pattern. For example, if as McCarley et al. (1997) proposed, schizophrenia is in part related to a failure of neuromodulation at (real) 
neuronal synapses, then one implication would be that a schizophrenic effect in semantic memory might be modeled by manipulating connection weights while holding constant other parametric values. We present such a model of schizophrenic associative disturbance below (Han et al., 2001), but first we describe the behavioral experiments that led to the simulation (Nestor et al., 1998).

Nelson and colleagues developed a network model of associative memory in which activation within a putative semantic structure or architecture is modulated by parametrically determined qualities of words. Both the size and the degree of connectivity of a network of a given word will determine the spread of activation within semantic space. Each of these dimensions has been quantitatively derived from extensive normative studies of word associations performed by Nelson and colleagues over the past 15 years. In fact, Nelson and colleagues have developed a database of 5019 words from more than 6000 participants from which network size and degree of connectivity for each of the words were computed. For example, as shown in Fig. 1, Nelson and colleagues computed the frequency of responses subjects made when asked to free associate to the word, THREAD. The normative studies demonstrated six associates to word THREAD, which in descending order of frequency were NEEDLE, SEW, STRING, CLOTH, CLOTHES, and ROPE. The number of associates represents the network size of the word, THREAD. The number of connections among associates represents the degree of connectivity of its network (Fig. 1).

Consider the example provided by Nelson and colleagues of the words DOG and DINNER. Each has five associates which include CAT, PUPPY, ANIMAL, HOUSE, and FRIEND for DOG, and SUPPER, EAT, LUNCH, FOOD, and MEAL for dinner. However, as Nelson et al. have shown, among the five associates of DOG, only one associate is connected to another associate (CAT). By contrast, results of normative studies by Nelson and colleagues revealed that DINNER has 17 connections among its associates. Each word, DOG and DINNER, therefore, have the same network size of five associates, but different degree of connectivity among associates. Nelson et al. computed a metric to capture the degree of connectivity of network of associates by dividing the number of connections among associates of a word by its network size. The connectivity index then equals $0.20(1 / 5)$ for the word DOG and 3.40 $(17 / 5)$ for the word DINNER. Normative studies have also suggested low correlation between network size and connectivity, suggesting that these factors may represent independent dimensions.

Using a connectionist framework, Nelson et al. developed a word recall paradigm to isolate the effects of network size and connectivity on associative memory in healthy subjects. As shown in Table 1, the paradigm consisted of four types of words: (1) high connectivity-small network (e.g. waves, wife, zoo), (2) low connectivity-small network (e.g. cap, clock, hive), (3) high connectivity-large network (e.g. bottle, cloth, flute), and (4) low connectivity-large network (e.g. blood, party, rabbit). Subjects first studied a list of words consisting of equal proportions of these four types, followed by a cued recall test in which word cues and target words are equated on both connectivity and network size. In several carefully controlled experiments, Nelson and colleagues have found support for their model: words of small networks are easier to recall than words of large networks, and words of high connectivity are easier to recall than words of low connectivity. In addition, for single-trial cued recall, performance is best for words of high connectivity-small network (e.g. wife cued by spouse) followed by low connectivity-small network (e.g. clock cued by time), then by high connectivity-large network (e.g. bottle cued by cork), and last by low connectivity-large network (e.g. party cued by birthday) (Table 1).

We have used this paradigm to isolate the effects of connectivity and network size on word recall in patients with schizophrenia (Nestor et al., 1998). Our prior neurophysiological studies 
have consistently demonstrated abnormalities in the semantically sensitive ERP, N400, which we have interpreted as evidence of aberrant semantic activation in schizophrenia (Adams et al., 1993; Nestor et al., 1997; Niznikiewicz et al., 1997). The question addressed in this behavioral study was whether such aberrant activation might be related to abnormalities in connectivity and/or network size within the lexicon of the schizophrenic brain. Eighteen patients with schizophrenia and aged-matched controls studied a list of 32 words of equal difficulty and controlled for frequency. The list contained equal proportions of the four types of words normed by Nelson and colleagues on the dimensions of connectivity and network size. A test of cued recall followed the presentation of the study list, with these experimental conditions identical to those used by Nelson and colleagues. Results indicated that schizophrenic patients recalled fewer words, and showed a particularly pronounced effect of the connectivity of the to-be-remembered word. For patients, regardless of network size, recall improved substantially for words of high connectivity and declined dramatically for words of low connectivity. By contrast, the comparison subjects showed the expected effects, with best recall for words of high connectivity-small network size, followed by words of low connectivity-small network size, then by words of high connectivity-large network size, and finally by words of low connectivity-large network size.

These findings thus extended our prior neurophysiological as well as neuroanatomical studies of semantic processing in schizophrenia by demonstrating a fairly selective, disease-related disturbance in connectivity. The data highlighted the potential significance of modulation of associative links, which in connectionist models is a function ascribed to attention that governs the spread of activation within the semantic/lexical system. The data raised specific questions as to whether the observed schizophrenic pattern of recall reflects a dysregulation of connections that bind associates within local semantic networks. To address these questions, two distinct but related levels of modeling are presented. In the first case, schizophrenic word recall is simulated using a simplified artificial network with connection weights extrapolated from norms provided by Nelson and colleagues. In the second case, we present an in vitro cellular model that provides biological evidence as to specific mechanisms that might modulate associative links, their neuropharmacological and temporal properties as well as their functional consequences in terms of signal processing and perceptual matching.

\section{Word association neural network}

A computer simulation known as the Word Association Neural Network (WANN) was constructed to model the cued recall of normal and schizophrenic subjects (Han et al., 2001) WANN was designed according to the word association neural network derived from the matrices of Nelson et al. (1998). The strength of the connection weights and the nodes of the neural network were easily represented respectively by the matrices' degrees of associations and the words themselves. The computer simulation was powered by the principles of parallel distributive processing as described by Rummelhart and McClelland (Rummelhart and McClelland 1986a,b). It modeled an empirically supported, psychobiologically viable process that allows for multiple computations to occur at once to achieve a desired outcome. In WANN, activation is entered at a node and proceeds to disperse according to the connection weights of that particular node until a steady state (no change) occurs in the entire system. Activation entering into the system from outside of the system is representative of a behavioral activity. The node with the highest activity apart from the cue word becomes the response. To illustrate, a word association neural network is shown below (Fig. 2):

The circles are the nodes that correspond behaviorally to representations of words. The arrows represent the direction and strength of the associations between the respective nodes (words). For example, the percent chance that a subject will respond with the target THREAD when given the cue NEEDLE is $42 \%$. Likewise, there is a $22 \%$ chance that the subject will respond 
with the word SEW. Both of these relationships are represented by the green connections in the figure. Hypothetically, if the word SEW is given as the cue, there is a $16 \%$ chance that a subject will respond with NEEDLE, a 14\% chance the subject will respond with THREAD, a $11 \%$ chance the subject will respond with CLOTHES, and a $3 \%$ chance the subject will respond with CLOTH (represented by blue connections in the figure). These chance percentages represented as probabilities in Fig. 2 are reflective of the matrix word response frequencies empirically derived by Nelson et al. (1998) and thus serve as the connection weights between nodes for WANN computer simulation (see Fig. 1 on page 10).

If the words are taken as unique and separate nodes, and if the associations can be mapped and weighted such as they are in Fig. 2, then this mapping thus allows for a computational correlate of Nelson's word associating exercise. A numerical activation can begin anywhere in the network, and that activation can disperse throughout the network according to the association relationships. To illustrate the activation and propagation process of the WANN, Fig. 3 and Fig. 4 are provided below. Fig. 3 illustrates the effect of studying the target word, and Fig. 4 illustrates the effect that the presentation of the cue word has upon the neural network after the study of the target word. The node with the highest activation other than the cue word is representative of the subject's response.

During the first set of iterations designed to simulate normal subjects, WANN revealed a pattern of recall that mimicked Nelson's results according to set size. Small set size words were recalled more often than large set size words. However, it did not reveal the same pattern of recall with respect to connectivity shown by Nelson et al. (1993). Words of lower connectivity were recalled higher than words of higher connectivity. Upon further analysis it was discovered that the effects of noise (a randomness implicated in most computer simulations of psychobiological processes) disproportionately compromised networks of higher connectivity. Since there is more competition for the correct target word among highly connective networks due to the prevalence and strength of connection weights, the recall for highly connective words was negatively affected. Two manipulations were successfully imposed upon the WANN to produce the normal subject pattern of recall. One varied the input activation with respect to connectivity and the other varied the noise with respect to connectivity. Below is the calculation for the 'weight input' manipulation:

$$
a^{\prime}=a \times(1+(c /(w / 100)))
$$

where $a^{\prime}$ is the amount of activation input into the node, $a$ is the amount of activation that would have been input into the node had 'weightinput' been ' 0 ', $c$ is the connectivity of the node, and $w$ is the value of 'weightinput'.

Below is the calculation for the 'weight noise' manipulation:

$$
e^{\prime}=e /(1+(c \times(w / 100)))
$$

where $e^{\prime}$ is the extent to which noise effects the node, $e$ is the extent to which noise would have affected the node had 'weightnoise' been ' 0 ', $c$ is the connectivity of the node, and $w$ is the value of 'weightnoise'

Hebbian theory may support both of these as neurobiologically representative manipulations. According to Nelson et al. (1993), highly connective words are recalled more due to a stronger surge of activation surrounding the target word. The findings of our study challenge this theoretical explanation for the greater prevalence of highly connective words being recalled, as well as Nelson et al.'s perception of connectivity. The recall of schizophrenic subjects was 
successfully simulated using the WANN by manipulating the connection weights and the amount of noise present in the neural network system. The manipulation of connection weights appears to be a fundamental prerequisite to the schizophrenic response.

\section{Neural circuit model of schizophrenia}

A key element of a network, whether it is cognitive, computational or biological, is its ability to maintain stability and to constrain excitation, typically in the service of informational selectivity. In the foregoing presentation of WANN, excitation spreads as a function of arbitrarily-set values of activation and parametrically-derived connections weights. These manipulations proved sufficient to model the connectivity-network size effect in normal subjects as well as the abnormal connectivity effect in schizophrenic patients. In real biological systems, however, excitation or activation by itself is insufficient, and almost always requires to be balanced by inhibition. This too is also common in many artificial networks of cognition, particularly in the cases of perception and selective attention (Walley and Weiden, 1973;

McClelland and Rummelheart, 1981). This balancing act, so to speak, is often modeled after a physiological process that is expressed at the cellular level of local neural circuits, known as recurrent inhibition.

Throughout the CNS, recurrent (feedback) and feed-forward inhibition represent the two major means by which activation and excitation are constrained. Each form has a different source of origination. Feed-forward inhibition originates extrinsically in brainstem and subcortical nuclei, whereas recurrent inhibition emanates intrinsically from collateral neurons of local excitatory projection cells. Whenever a projection neuron is activated, a local recurrent circuit will fire back on the projection neuron, reducing the spread of excitation emanating from the projection neuron. Moreover, the firings of both projection and recurrent neurons overlap both spatially and temporally, resulting in the modulation of both lateral spread and duration of firing of excitatory projection neurons. Recurrent inhibitory circuits are thought to be highly represented in cortical networks known for having pronounced excitatory interactions, such as the medial temporal lobe, particularly the hippocampus.

From their in vitro studies of the CAI field of the rat hippocampus, McCarley et al. (1999) have developed a neural circuit model that identifies faulty recurrent inhibition as an important cellular event in the pathogenesis of schizophrenia. As shown in Fig. 5, the architecture of the model consists of a simplified recurrent inhibition circuit with projection and target neurons mediated by inhibitory inter-neurons. In their studies of the CA1 region of the hippocampus in vitro, McCarley et al. showed that glutamatergic projection neurons excite inhibitory GABAergic inter-neurons, which in turn back propagate inhibition on projection neurons. This recursive inhibitory feedback produced an inhibitory post-synaptic potential (IPSP), which originated from NMDA receptor activation of the glutamatergic projection neuron (Grunze et al., 1996). Moreover, Gunze at al demonstrated that in response to a tetanizing stimulus, the intracellularly recorded recurrent IPSP produced long term potentiation (LTP), the sine qua nom of cellular learning and memory. Grunze and colleagues further demonstrated the LTP of the recurrent inhibition circuit to be differentially sensitive to blockade by NMDA antagonist APV. In fact, when compared to excitatory LTP produced by stimulation of excitatory input (Schaffer collateral), the LTP of IPSP proved to be 10-fold more sensitive to blockade by NMDA antagonists.

The neural circuit model conforms well with what is generally known about the neurobiology of schizophrenic cognition. First, these NMDA circuits are highly represented in brain areas, such as the medial temporal lobe, which are frequently implicated in schizophrenia. Second, that recurrent inhibition circuit proved to be differentially sensitive to NMDA blockage would be consistent with the importance of these antagonists in drug models of schizophrenia 
(McCarley et al., 1999). Third, in the proposed circuit model, reduction of recurrent inhibition produced over activation of projection neurons, resulting in possible excitotoxic damage to target neurons. The extent to which such poorly constrained cellular activation resembles the proposed aberrant semantic activation in schizophrenia is of course unknown. However, the findings from these in vitro studies did conform well to the hypothesis of poorly constrained semantic activation and faulty inhibition of associative links as important cognitive events in schizophrenic cognition.

In order to pursue further the link between cellular recurrent inhibition and schizophrenic cognition, Grunze and colleagues performed a biophysical simulation of the processing characteristics of CAI neuronal circuitry. Pattern discrimination and recall was simulated by strengthening synapses in the network according to a Hebbian rule. During the learning phase, both pre- and post-synaptic excitatory activity received excitatory LTP. The network subsequently learned to discriminate and to store two different but overlapping patterns of afferent input, one with a complete array of elements and the other with a reduced array of the same elements. Each of the two patterns activated 40 neurons with eight of these neurons common to both complete and degraded input patterns. During the test phase, the network recalled specific patterns of action potential generation in modeled neurons. For example, when presented with a degraded input pattern, the network characteristically responded with 24 of the original 40 neurons activated by the complete input.

Grunze et al. (1996) also observed, however, that while the network recalled missing elements of stored patterns, errors of discrimination between similar patterns increased. As they noted: "The capability to recall missing elements of the stored pattern also enhanced the possibility that activity elicited by one pattern would spread into neurons activated by the other pattern, thus reducing or preventing the ability to differentiate between the patterns" (p. 2039-2040). By strengthening the excitatory synapses onto inhibitory inter-neurons, in essence producing recurrent inhibition, led to the expected effect of constraining activation and the ability to discriminate overlapping stored patterns. Without strengthening recurrent inhibition during recall, action potentials diffused across neurons representing both patterns, preventing the ability to distinguish between overlapping stored patterns.

\section{Discussion}

We began with a review of the biology of attention and semantic processes as studied by cognitive neuroscience. Attention here was conceptualized as a finite, limited capacity that is an intrinsic byproduct of the sensory, perceptual and cognitive machinery of the brain. Attention effects are expressed across a number of levels of brain organization from local circuits to wide scale representational systems. The effects evident at the circuit level are one of modulation resulting in either selective enhancement or inhibition of neural output. Attention interacts with lexical-semantic structures directing and governing activation within a lexicon distributed across frontal and temporal lobes. Efficient and healthy cognition depends in large part on activation-reducing mechanisms allowing for the resolution among competing representations (see Anderson and Spellman, 1995).

Schizophrenic thought disorder is hypothesized to reflect faulty interaction between these attention and semantic structures. An attention modulation disturbance in associative links that bind words within the lexicon is thought to produce abnormal connectivity effects demonstrated on word recall by patients with schizophrenia. Here the behavioral effect would appear to fit best with a modulation failure of attention, with schizophrenic performance characterized by over-responding to words of high connectivity and under-responding to words of low connectivity. In fact, we have recently begun computer simulation studies of cued-recall performance using words of varying degrees of connectivity and network size. Our results have 
modeled the normal effect of better performance for words of high connectivity-small network, pointing to the importance of these word features in cued retrieval from semantic memory. In addition, by 'lesioning' connection weights, while holding constant network size, the model effectively demonstrated cued recall performance similar to that observed in behavioral studies of schizophrenic patients.

What might be the causal biological mechanisms and how might they be characterized? In other words what might be the important empirical details of the hypothesized faulty interaction of attention and semantic structures and how might they be tested in schizophrenic patients?

To address these questions, we looked to results of biophysical simulation of real networks of neurons with identifiable pharmacological properties and processing characteristics, and which are highly represented in cortical areas that have been implicated in schizophrenic neuropathology. These simulation results have provided fairly strong biological evidence of the importance of NMDA mediated recurrent inhibition in distinguishing pairs of overlapping patterns of afferent input by the modeled network of the CA1 field in the rat hippocampus. Even more interesting, however, were the findings that emerged when reducing recurrent inhibition by NMDA receptor blockade. The ensuing aberrant spread of neuronal excitation as a result of reduced recurrent inhibition led to discrimination errors, with confusion of previously distinguishable input patterns.

Recurrent inhibition provides a vehicle to unify levels of psychological description with identifiable physical events. Indeed, its cognitive analogue often referred to as lateral inhibition has been ascribed an important role in resolving competition and selection in perception, selective attention, and memory retrieval (McClelland and Rummelheart, 1981; Anderson and Spellman, 1995). For schizophrenic thought disorder, evidence ranging from biophysical to psychological all points to the potential role that failed recurrent inhibition might play in the disease-related disturbance in cognition. Emerging from these data is a testable hypothesis of failed recurrent inhibition in schizophrenia. Stated simply, the hypothesis proposes that a failure in NMDA mediated recurrent inhibition leads to aberrant spread of activation throughout the schizophrenic brain, especially evident in temporal regions that have been known to be both rich in NMDA receptors and to be intimately linked to lexical and semantic structures and processes. What ensues is a hypothesized failure of modulation of putative connection weights that bind word associates within local networks. Thus, in this proposed model, failed recurrent inhibition is hypothesized as a biological substrate for a set of cellular events that leads to the abnormal connectivity effect in word recall by patients with schizophrenia.

The effects of failed recurrent inhibition in schizophrenia might be evident relatively early in the information processing stream long before controlled, voluntary effortful, strategic attention processes are engaged. Moreover, such a relatively temporally early occurring disturbance would be more aligned with a problem with attention processes that are generally described as fast-acting, automatic, involuntary, obligatory, and effortless, and typically do not include inhibitory functions that are commonly characterized under the rubric of controlled processes. Information processing studies in schizophrenia have often attributed disease related disturbances to so-called controlled but not automatic processes of attention. However, Nestor and O'Donnell (1997) reviewed information processing studies in schizophrenia that pointed to disease related disturbances in processes that presumably occur temporally early without awareness, and are independent of controlled effortful attention. For example, backward masking deficits are greatest in schizophrenic patients with a mask following the target between 20 to $70 \mathrm{~ms}$. Similarly, using a flanking paradigm, Elkins and Cromwell (1994) demonstrated that flankers incompatible with targets produced significantly less interference for schizophrenic patients than for normal controls and depressed patients. Noting that the effect occurred for SOAs of no more than $200 \mathrm{~ms}$, Elkins and Cromwell interpreted reduced 
interference in schizophrenic subjects as evidence of a disease-related disturbance in fastacting, automatic attention different from the strategic, controlled inhibition.

Recurrent inhibition might not only selectively enhance signal processing of neural circuits, but also promote synchronous oscillations of temporally linked neurons (e.g. Niebur and Koch, 1997). Such synchronous firing, specifically within the gamma range (30 to $50 \mathrm{~Hz}$ ), has been frequently proposed as a key information processing mechanism of neural networks underlying perceptual and temporal binding (e.g. Shastri and Ajjanagadde, 1993; Joliot et al., 1994; Singer and Gray, 1995; Niebur and Koch 1997). Gamma synchronization might also be dependent upon the interplay between GABA and excitatory amino acid transmission, specifically the NMDA receptor, abnormalities of which have been frequently implicated in information processing disturbances as well in schizophrenia (Coyle, 1996; McCarley et al., 1996; Traub et al., 1996). Not surprisingly then, gamma abnormalities have recently been investigated in schizophrenia by our laboratory (Kwon et al., 1999). We evaluated the power and phase of auditory frequency following in the EEG during entrainment to auditory clicks at varying frequencies $(20,30$, and $40 \mathrm{~Hz})$. The results indicated reduced power for 40 but not 20 or 30 $\mathrm{Hz}$ in patients with schizophrenia in relation to control subjects. In addition, while control subjects showed early onset of entrainment to $40 \mathrm{~Hz}$ stimuli, schizophrenic patients showed delayed onset of entrainment, poorer synchronization, and a longer persistence of entrainment after the end of the $40 \mathrm{~Hz}$ string of auditory clicks. These findings suggested patients with schizophrenia have decreased synchronous activity of neuronal firings at $40 \mathrm{~Hz}$ but not lower frequencies.

Gamma band oscillations may also play an important role in the processing of lexical and semantic representations. Lisman (1998), for example, has suggested that $\gamma$ band oscillations function to separate different units of information so that each discrete $40 \mathrm{~Hz}$ cycle represents a distinct item of semantic memory. As evidence, Lisman cited studies showing that nested within the slower $5-\mathrm{Hz}$ 'theta' oscillation of hippocampal and related cortical area recordings are approximately seven cycles of $40 \mathrm{~Hz}$ (Bragin et al., 1995). These seven cycles correspond to the well-known capacity limits of short term memory (Lisman and Idiart, 1995). In addition, Lisman (1998) noted that scanning through each additional new item held in short term memory requires an amount of time that approximates the $40-\mathrm{Hz}$ period. Also relevant are findings of Pulvermuller and colleagues (Lutzenberger et al., 1994; Pulvermuller, 1996) that have demonstrated differential gamma band $(30 \mathrm{~Hz})$ power for words in relation to pseudo-words in healthy subjects. Thus, taken together, these data suggest that gamma functions not only to bind percepts but also to keep distinct memories and other presumably related lexical entries. These data parallel the findings of Grunze et al. (1996) that failed gamma due NMDA blockade of recurrent inhibition led to reduced discriminability of percepts with overlapping elements. Of course, the extent, if any, to which schizophrenic gamma failure may contribute to the disease-related word association disturbance has yet to be established. Recent preliminary data, however, has demonstrated abnormal $30 \mathrm{~Hz}$ gamma for words presented in a semantic priming paradigm in schizotypal subjects (Niznikiewicz et al., 1997).

In conclusion, the proposed model is intended to serve as a heuristic device to organize data from often disparate sources so that testable hypotheses might be developed that will eventually unify biological and psychological levels of analyses of schizophrenic cognition. It remains to be seen the extent, if any, to which schizophrenic abnormalities in connectivity in word recall paradigms might be related to those observed in gamma in EGG studies, and how both might originate from an NMDA mediated cellular failure of recurrent inhibition. However, the important point to underscore is that the proposed model describes a conceptual and experimental framework to explore, test, and falsify the hypothesis of failed recurrent inhibition as the biological mechanism by which attention and semantic interact to produce schizophrenic cognition. 


\section{Acknowledgments}

Supported by MERIT Awards from the Department of Veterans Affairs Medical Research Service (Dr McCarley and Dr Shenton), the Department of veterans Affairs Schizophrenia Center Grant (Dr McCarley), NIMH grants MH-50740, MH-01110 (Dr Shenton), and MH-40799 (Dr McCarley), and Young Investigator Award to Dr Nestor from the National Alliance for Research on Schizophrenia and Depression.

\section{References}

Adams J, Faux SF, Nestor PG, et al. ERP abnormalities during semantic processing in schizophrenia. Schizophr. Res 1993;10:247-257. [PubMed: 8260443]

Anderson MC, Spellman BA. On the status of inhibitory mechanisms in cognition: memory retrieval as a model case. Psychol. Rev 1995;102:68-100. [PubMed: 7878163]

Barch DM, Cohen JD, Servan-Schreiber D, Steingard S, Cohen JD, Steinhauer SS, van Kammen DP. Sematic priming in schizophrenia and examination of spreading activation using word pronounciation and multiple SOAs. J. Abnorm. Psychol 1996;105:592-601. [PubMed: 8952192]

Beech AR, Clarridge GS. Individual differences in negative priming: relations with schizotypal personality traits. Br. J. Psychol 1987;78:349-356. [PubMed: 3620793]

Beech AR, Powell J, McWilliam J, Claridge GS. Evidence of reduced 'cognitive inhibition' in schizophrenia. Br. J. Clin. Psychol 1989;28:109-116. [PubMed: 2743051]

Besner D, Smith MC, MacLeod CM. Visual word recognition: a dissociation of lexical and semantic processing. J. Exp. Psychol. Learn. Mem. Cogn 1990;16:832-869.

Bragin A, Jando G, Nadasdy Z, Hetke J, Wise K, Buzsaki G. Gamma (40-100 Hz) oscillations in the hippocampus of the behaving rat. J. Neurosci 1995;15(171):47-60. [PubMed: 7823151]

Britten KH. Attention is everywhere. Nature 1996;382:497-498. [PubMed: 8700219]

Callaway E, Naghdi S. An information processing model for schizophrenia. Arch. Gen. Psychiatry 1982;39:339-347. [PubMed: 7065844]

Carmazza A. The brains dictionary. Nature 1996;380:485-486. [PubMed: 8606763]

Chapman, LJ.; Chapman, JP. Disordered Thought In Schizophrenia. Englewood Cliffs, NJ: PrenticeHall; 1973.

Cohen JD, Servan-Schreiber D. Context, cortex, and dopamine. A connectionist approach to behavior and biology in schizophrenia. Psychol. Rev 1992;99:45-77. [PubMed: 1546118]

Colby CL. The neuroanatomy and neurophysiology of attention. J. Child Neurol 1991;6:S90-S118. [PubMed: 1848251]

Collins AM, Loftus EF. A spreading-activation theory of semantic processing. Psychol. Rev 1975;82:407-428.

Corbetta M, Miezin FM, Dobmeyer S, Shulman GL, Peterson SE. Selective and divided attention during visual discrimination of shape, color, and speed: functional anatomy by positron emission tomography. J. Neurosci 1991;11:2383-2402. [PubMed: 1869921]

Coyle JT. The glutamatergic dysfunction hypothesis for schizophrenia. Harv. Rev. Psychiatry 1996;3:241-253. [PubMed: 9384954]

Craik FI, Tulving E. Depth of processing and the retention of words in episodic memory. J. Exp. Psychol. Gen 1975;104:268-294.

Crick F, Koch C. Towards a neurobiological theory of consciousness. Semin. Neurosci 1990;2:263-275.

Damassio H, Grabowski TJ, Tranel D, Hichwa RD, Damasio AR. A neural basis for lexical retrieval. Nature 1996;380:499-505. [PubMed: 8606767]

Elkins IJ, Cromwell RL. Priming effects in schizophrenia: associative interference and facilitation as a function of visual context. J. Abnorm. Psychol 1994;103:791-800. [PubMed: 7822582]

Firth CD. Consciousness, information processing and schizophrenia. Br. J. Psychiatry 1979;134:225235. [PubMed: 509004]

Fischler, I. Attention and language. In: Parasuraman, R., editor. The Attentive Brain. Cambridge, MA: MIT Press; 1997. p. 381-400.

Grunze HCR, Rainnie DG, Hasselmo ME, et al. NMDA-dependent modulation of CA1 local circuit inhibition. J. Neurosci 1996;16:2034-2043. [PubMed: 8604048] 
Han SD, Nestor PG, Hannah GJ, Niznikiewicz MA, McCarley RW. Word association neural network: computer simulation of recall in control and schizophrenic subjects (under review). 2001

Haxby JV, Grady CL, Horwitz B, et al. Dissociation of object and spatial visual processing pathways in human extrastriate cortex. Proc. Natl. Acad. Sci. USA 1991;88:1621-1625. [PubMed: 2000370]

den Heyer K, Briand K, Dennenbring G. Strategic factors in a lexical decision task: evidence for automatic and attention-driven processes. Mem. Cogn 1983;11:374-381.

Hillyard, SA.; Mangun, GR.; Woldorff, MG.; Luck, SJ. Neural systems mediating selective attention. In: Gazzaniga, MS., editor. The Cognitive Neurosciences. Cambridge, MA: MIT Press; 1995.

Johnston, MH.; Holzman, PS. Assessing Schizophrenic Thinking: a Clinical and Research Instrument for Measuring Thought Disorder. San Francisco, CA: Jossey-Bass; 1979.

Joliot M, Ribary U, Llinas R. Human oscillartory brain activity near $40 \mathrm{~Hz}$ coexists with cognitive temporal binding. Proc. Natl. Acad. Sci. USA 1994;91:11748-11751. [PubMed: 7972135]

Just MA, Carpenter PA, Keller TA, et al. Brain activation modulated by sentence comprehension. Science 1996;274:114-116. [PubMed: 8810246]

Kwapil TR, Hegley DC, Chapman LJ, Chapman JP. Facilitation of word recognition by semantic priming in schizophrenia. J. Abnorm. Psychol 1990;99(3):215-221. [PubMed: 2212270]

Kwon J-S, O’Donnell BF, Wallenstein GV, et al. Gamma frequency range abnormalities to auditory stimulation in schizophrenia. Arch. Gen. Psychiatry 1999;56:1001-1005. [PubMed: 10565499]

Lisman J. What makes the brain's ticker tock. Nature 1998;394:132-133. [PubMed: 9671294]

Lisman JE, Idiart MA. Storage of $7 \pm 2$ short-term memories in oscillatory subjects. Science 1995;267:1512-1515. [PubMed: 7878473]

Lutzenberger W, Pulvermuller F, Birbaumer N. Words and pseudowords elicit distinct patterns of 30-Hz EEG responses in humans. Neurosci. Lett 1994;18:115-118. [PubMed: 7970225]

Manschreck TC, Maher BA, Milavetz JJ, Ames D, Weisstein CC, Schneyer ML. Semantic priming in thought disordered schizophrenic patients. Schizophr. Res 1988;1:61-66. [PubMed: 3154508]

McCarley RW, Shenton ME, O'Donnell BF, et al. Auditory P300 abnormalities and left posterior temporal gyrus volume reduction in schizophrenia. Arch. Gen. Psychiatry 1993;50:190-197. [PubMed: 8439239]

McCarley RW, Hsiao J, Freedman R, Pfefferbaum A, Donchin E. Neuroimaging and the cognitive neuroscience of schizophrenia. Schizophr. Bull 1996;22:703-726. [PubMed: 8938923]

McCarley RW, O’Donnell BF, Niznikiewicz MA, et al. Update on electrophysiology of schizophrenia. Int. Rev. Psychiatry 1997;9:373-386.

McCarley RW, Niznikiewicz MA, Salisbury DF, et al. Cognitive dysfunction in schizophrenia: unifying basic research and clinical aspects. Eur. Arch. Psychiatry Clin. Neurosci 1999;249:69-82. [PubMed: 10654112]

McClelland JL, Rummelheart DE. An interactive activation model of context effects in letter perception: part 1. An account of basic findings. Psychol. Rev 1981;88:375-407.

Mesulam M. Large-scale neurocognitive networks and distributed processing for attention, language, and memory. Ann. Neurol 1990;28:597-613. [PubMed: 2260847]

Moran J, Desimone R. Selective attention gates visual processing in the extrastriate cortex. Science 1985;229:782-784. [PubMed: 4023713]

Neely JH. Semantic priming and retrieval from lexical memory: evidence for facilitatory and inhibitory processes. Mem. Cogn 1976;4:648-654.

Neely JH. Semantic priming and retrieval from lexical memory: roles of inhibitionless spreading activation and limited-capacity attention. J. Exp. Psychol. Gen 1977;106:226-254.

Neill WT, Valdes LA. Persistence of negative priming: steady state or decay? J. Exp. Psychol. Learn. Mem. Cogn 1992;18:565-576.

Nelson DL, Bennett DJ, Gee NR, Schreiber TA, McKinney VM. Implicit memory: effects of network size and interconnectivity on cued recall. J. Exp. Psychol 1993;19:747-764.

Nelson DL, McEnvoy CL, Schreiber TA. The University of South Florida word association, rhyme, and word fragment norms. 1998

Nestor, PG.; O’Donnell, BF. The mind adrift: attentional dysregulation in schizophrenia. In: Parasuraman, R., editor. The Attentive Brain. Cambridge, MA: MIT Press; 1997. p. 527-546. 
Nestor PG, Kimble MW, O’Donnell BF, et al. Aberrant semantic activation in schizophrenia: a neurophysiological study. Am. J. Psychiatry 1997;154:640-646. [PubMed: 9137119]

Nestor PG, Akdag SJ, O’Donnell BF, et al. Word recall in schizophrenia: a connectionist model. Am. J. Psychiatry 1998;155:1685-1690. [PubMed: 9842776]

Niebur, E.; Koch, C. Computational architectures for attention. In: Parasuraman, R., editor. The Attentive Brain. Cambridge, MA: MIT Press; 1997. p. 163-186.

Niebur E, Koch C, Rosin C. An oscillation-based model for the neuronal basis of attention. Vis. Res 1993;33:2789-2802. [PubMed: 8296473]

Niznikiewicz MA, O’Donnell BF, Nestor PG, et al. ERP assessment of visual and auditory language processing in schizophrenia. J. Abnorm. Psychol 1997;106:85-94. [PubMed: 9103720]

Nobre AC, McCarthy G. Language-related ERP's: scalp distributions and modulation by word type and semantic priming. J. Cogn. Neurosci 1994;6:233-255.

Parasuraman, R. The attentive brain: issues and prospects. In: Parasuraman, R., editor. The Attentive Brain. Cambridge, MA: MIT Press; 1997. p. 3-16.

Posner, MI. Structures and functions of selective attention. In: Boll, T.; Bryant, B., editors. Master Lectures in Clinical Neuropsychology and Brain Function: Research, Measurement, and Practice. Washington, DC: American Psychological Association; 1988. p. 171-202.

Posner, MI.; Snyder, CRR. Facilitation and inhibition in the processing of signals. In: Solsa, RL., editor. Attention and Performance V. New York: Academic Press; 1975.

Pulvermuller F. Hebb's concept of cell assemblies and the psychophysiology of word processing. Psychophysiology 1996;33:317-333. [PubMed: 8753932]

Ramachadran VS, Cobb S. Visual attention modulates metacontrast masking. Nature 1995;373:66-68. [PubMed: 7800040]

Rees G, Frackowiak R, Frith C. Two modulatory effects of attention that mediate object categorization in human cortex. Science 1997;275:835-838. [PubMed: 9012351]

Robbins, TW. Arousal and attention: psychopharmacological and neuropsychological studies in experimental animals. In: Parasuraman, R., editor. The Attentive Brain. Cambridge, MA: MIT Press; 1997. p. 189-220.

Rummelhart, DE.; McClelland, JL. Parallel Distributed Processing. Vol. vol. 1. Cambridge, MA: MIT Press; 1986a.

Rummelhart, DE.; McClelland, JL. Parallel Distributed Processing. Vol. vol. 2. Cambridge, MA: MIT Press; 1986b.

Salo R, Robertson LC, Nordahl TE, Kraft LW. The effects of antipsychotic medication on sequential inhibitory processes. J. Abnorm. Psychol 1997;106:639-643. [PubMed: 9358694]

Shastri L, Ajjanagadde V. From simple associations to systematic reasoning: a connectionist representation of rules, variables, and dynamic bindings using temporal synchrony. Behav. Brain Sci 1993;16:417-494.

Shenton ME, Kikinis R, Jolesz FA, et al. Abnormalities of the left temporal lobe and thought disorder in schizophrenia: a quantitative magnetic resonance imaging study. New Engl. J. Med 1992;327:604612. [PubMed: 1640954]

Singer W, Gray CM. Visual feature integration and the temporal correlation hypothesis. Annu. Rev. Neurosci 1995;18:555-586. [PubMed: 7605074]

Spitzer H, Desimone R, Moran J. Increased attention enhances both behavioural and neuronal performance. Science 1988;240:338-340. [PubMed: 3353728]

Spitzer M, Braun U, Maier S, Hermle L, Maher BA. Indirect semantic priming in schizophrenic patients. Schizophr. Res 1993;11:71-80. [PubMed: 8297807]

Spitzer M, Weisker I, Winter M, et al. Semantic and phonological priming in schizophrenia. J. Abnorm. Psychol 1994;103:485-493. [PubMed: 7930048]

Traub RD, Whittington MA, Collin SB, Buzsaki G. Analysis of gamma rhythms in the rat hippocampus in vitro and in vivo. J. Physiol 1996;493:471-484. [PubMed: 8782110]

Treue S, Maunsell JHR. Attentional modulation of visual motion processing in cortical areas. Nature 1996;382:539-541. [PubMed: 8700227] 
Walley RE, Weiden TD. Lateral inhibition and cognitive masking: a neuropsychological theory of attention. Psychol. Rev 1973;80:284-302. [PubMed: 4352821]

Williams GM, Mathews A, MacLeod C. The emotional Stroop task and psychopathology. Psychol. Bull 1996;120:3-24. [PubMed: 8711015]

Yee PL. Semantic inhibition of ignored words during a figure classification task. Q. J. Exp. Psychol. A 1991;43:127-153. [PubMed: 2017571] 


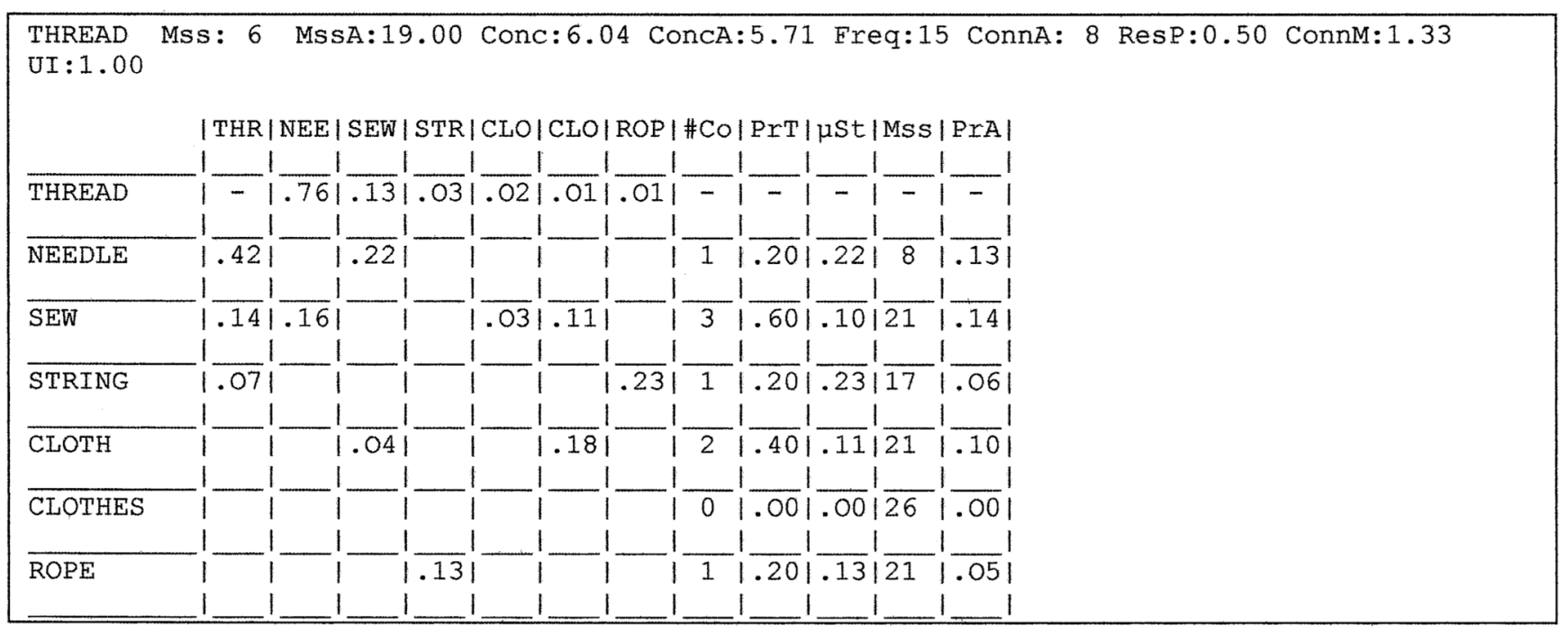

Fig. 1.

Free associate responses to the word, THREAD produced in descending order of frequency, NEEDLE, SEW, STRING, CLOTH, CLOTHES, and ROPE. 


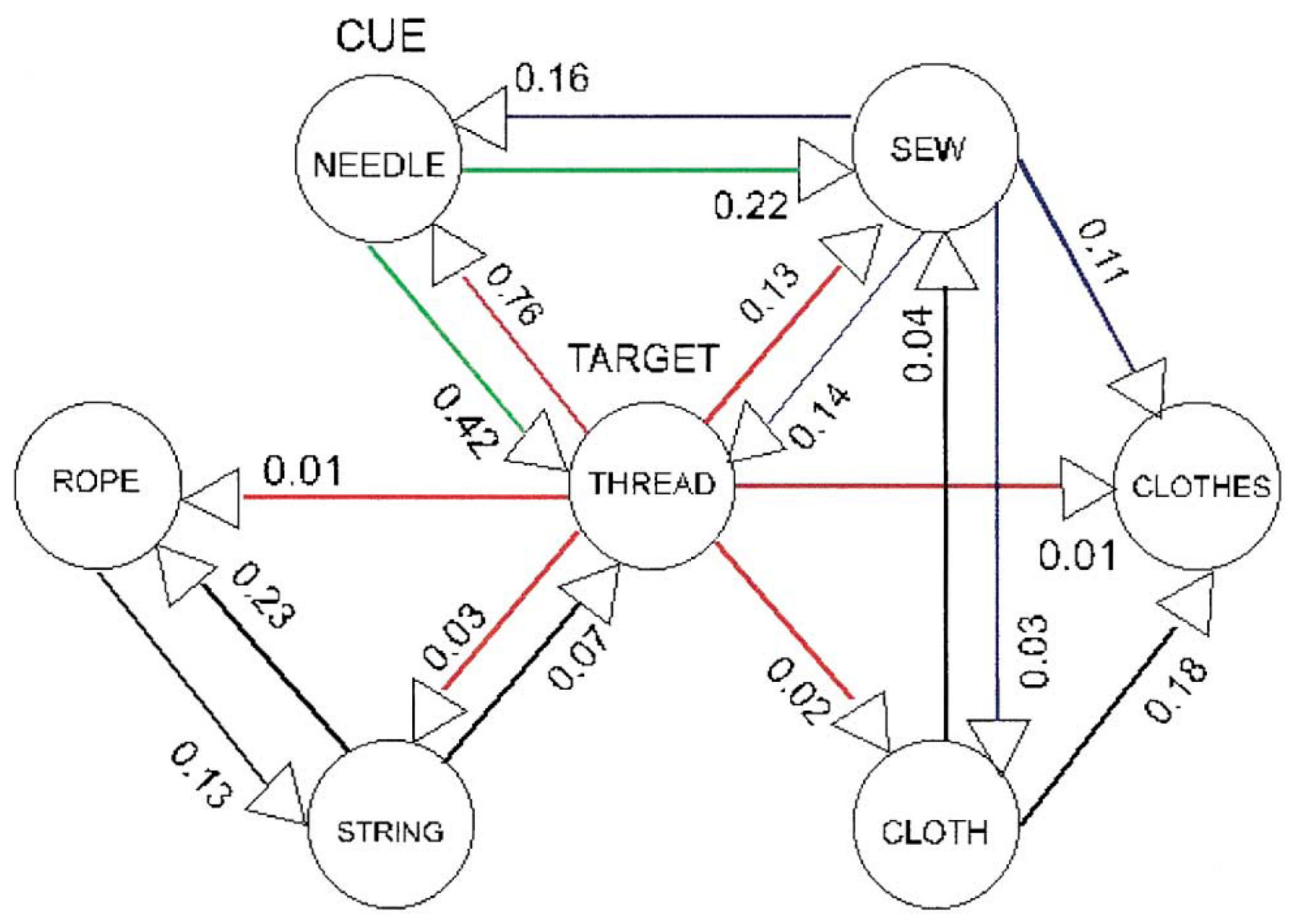

Fig. 2.

Schematic of basic network architecture of WANN (see text for further discussion). 


\begin{tabular}{|c|c|c|c|c|c|c|c|}
\hline \multicolumn{8}{|c|}{ Activate study word 'THREAD': } \\
\hline Units: & Time01: & Time02: & Time03: & Time04: & Time05: & $\rightarrow$ & Time22: \\
\hline THREAD & 100.00 & 100.00 & 133.95 & 137.18 & 149.91 & & 162.73 \\
\hline NEEDLE & 0.00 & 76.00 & 78.08 & 106.57 & 109.81 & & 131.72 \\
\hline SEW & 0.00 & 13.00 & 29.80 & 34.69 & 41.42 & & 50.32 \\
\hline STRING & 0.00 & 3.00 & 3.13 & 4.24 & 4.38 & & 5.25 \\
\hline CLOTH & 0.00 & 2.00 & 2.39 & 3.57 & 3.78 & & 4.76 \\
\hline CLOTHES & 0.00 & 1.00 & 2.79 & 5.05 & 5.83 & & 8.02 \\
\hline ROPE & 0.00 & 1.00 & 1.69 & 2.06 & 2.35 & & 2.83 \\
\hline
\end{tabular}

Fig. 3.

WANN propagation in response to studying target word. 


\begin{tabular}{|c|c|c|c|c|c|c|c|}
\hline \multicolumn{8}{|c|}{ Activate cue word 'NEEDLE': } \\
\hline Units: & Time23: & Time24: & Time25: & Time26: & Time27: & $\rightarrow$ & Time31: \\
\hline THREAD & 162.73 & 204.73 & 207.81 & 218.44 & 219.23 & & 219.48 \\
\hline NEEDLE & 231.72 & 231.72 & 255.00 & 255.00 & 255.00 & & 255.00 \\
\hline SEW & 50.32 & 72.32 & 77.78 & 83.36 & 84.75 & & 84.91 \\
\hline STRING & 5.25 & 5.25 & 6.51 & 6.66 & 7.02 & & 7.08 \\
\hline CLOTH & 4.76 & 4.76 & 6.26 & 6.49 & 6.87 & & 6.94 \\
\hline CLOTHES & 8.02 & 8.02 & 10.86 & 11.76 & 12.52 & & 12.78 \\
\hline ROPE & 2.83 & 2.83 & 3.25 & 3.58 & 3.72 & & 3.82 \\
\hline
\end{tabular}

Fig. 4.

WANN propagation in response to cued recall of target word. 


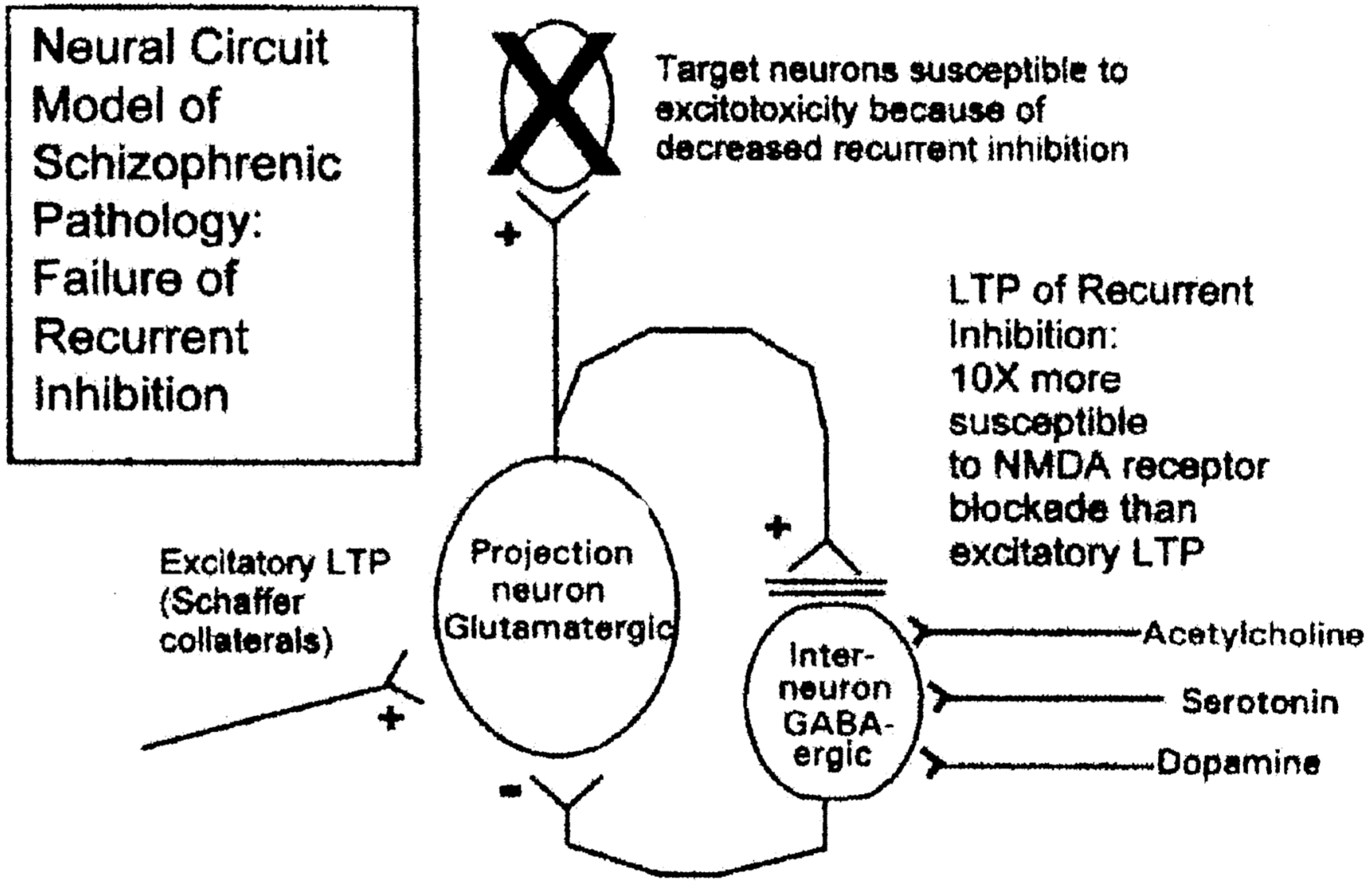

Fig. 5.

Cellular model of failure of recurrent inhibition, + is excitatory (glutamatergic) and - is inhibitory (GABA-ergic). The two horizontal lines on the projection neuron synapse represent NMDA receptor blockade. Note that inhibitory interneuron also has cholinergic, serotonergic, and dopaminergic modulatory synapses. These are also possible sites of abnormal receptors and/or abnormal inputs (see text for further discussion). 
Table 1

Example of words used in connectionist study (Nestor et al., 1998)

\begin{tabular}{lll}
\hline Connectivity and network size & Test cue & Target \\
\hline High connectivity-small network & Originate & Begin \\
& Surf & Waves \\
& Monkey & Zoo \\
Low connectivity-small network & Spouse & Wife \\
& Shower & Cap \\
& Time & Clock \\
High connectivity-large network & Bee & Hive \\
& Thumb & Nail \\
& Cork & Bottle \\
& Fabric & Cloth \\
Low connectivity-large network & Clarinet & Flute \\
& Whiskey & Drunk \\
& Cut & Blood \\
& Birthday & Party \\
& Gate & Fence \\
\hline
\end{tabular}

